\title{
Pigment epithelium-derived factor has a role in the progression of papillary thyroid carcinoma by affecting the HIF1 $\alpha$-VEGF signaling pathway
}

\author{
YICHEN LV ${ }^{1}$, YU SUN ${ }^{1}$, TIEFENG SHI ${ }^{1}$, CHENLEI SHI ${ }^{1},{\text { HUADONG } \text { QIN }^{1} \text { and ZHAOZHU LI }}^{2}$ \\ ${ }^{1}$ The Fourth Department of General Surgery; ${ }^{2}$ Department of Pediatric Surgery, \\ The Second Affiliated Hospital of Harbin Medical University, Harbin, Heilongjiang 150086, P.R. China
}

Received July 9, 2015; Accepted September 22, 2016

DOI: $10.3892 / \mathrm{ol} .2016 .5316$

\begin{abstract}
The progression mechanism of papillary thyroid carcinoma (PTC) remains largely unknown. Accumulating evidence has suggested that various targets of pigment epithelium-derived factor (PEDF) are able to inhibit cancer progression. The aim of the present study was to examine PEDF expression in PTC patients and to investigate its relationship with aggressive clinicopathological features, as well as to explore whether PEDF affects the progression of PTC via the hypoxia-inducible factor $1 \alpha$ (HIF1 $\alpha$ )-vascular endothelial growth factor (VEGF) pathway. A total of 271 patients with PTC, including 24 men and 247 women, were enrolled in the present study. Relevant patient data, including demographic features, preoperative clinical features and pathological features, were collected for analysis. The protein expression levels of PEDF in PTC tissues were detected using immunohistochemical staining, and the mRNA expression levels of PEDF, VEGF and HIF1 $\alpha$ in 15 PTC tissues with lymph node metastasis (LNM) and 10 tissues without LNM were detected using reverse transcription-quantitative polymerase chain reaction. Immunohistochemical staining with an anti-PEDF antibody detected PEDF expression in $74.5 \%$ of the PTC tissues. PEDF expression levels were significantly correlated with LNM, extrathyroid invasion, a high TNM stage, the presence of the $\mathrm{BRAF}^{\mathrm{V} 600 \mathrm{E}}$ mutation and tumor size. PEDF mRNA expression levels were significantly decreased in PTC tissues with LNM, as compared with PTC tissues without LNM,
\end{abstract}

Correspondence to: Professor Huadong Qin, The Fourth Department of General Surgery, The Second Affiliated Hospital of Harbin Medical University, 142 Baojian Road, Harbin, Heilongjiang 150086, P.R. China

E-mail: hdqin77@126.com

Professor Zhaozhu Li, Department of Pediatric Surgery, The Second Affiliated Hospital of Harbin Medical University, 142 Baojian Road, Harbin, Heilongjiang 150086, P.R. China

E-mail: 1zzhyd@126.com

Key words: papillary thyroid carcinoma, metastasis, pigment epithelium-derived factor, vascular endothelial growth factor, hypoxia-inducible factor $1 \alpha$ while the mRNA expression levels of HIFl $\alpha$ and VEGF were markedly increased in PTC tissues with LNM. Taken together, the results of the present study suggested that PEDF plays a role in the progression of PTC, and that PEDF may exert an anti-angiogenesis role by affecting the HIF1 $\alpha$-VEGF pathway, eventually inhibiting the metastasis of PTC.

\section{Introduction}

Thyroid cancer is the most common endocrine malignancy and papillary thyroid carcinoma (PTC) accounts for $80-90 \%$ of all thyroid malignancies $(1,2)$. The incidence of PTC has increased in most countries in the last three decades $(3,4)$. Although the majority of patients with PTC have a favorable prognosis following appropriate treatments, $10-30 \%$ of patients with PTC progress to metastasis or recurrence, and mortality in $5 \%$ of cases $(5,6)$. The mechanisms underlying the progression of PTC have yet to be elucidated.

For the majority of patients with PTC, metastasis remains the main cause of mortality; $>90 \%$ of cancer-associated mortalities are caused by tumor invasion and metastasis, which emphasizes the importance of elucidating the mechanisms underlying metastasis (7-9). The contribution of angiogenesis to tumor progression is well established. In human solid cancer, the growth and survival of tumor cells are angiogenesis-dependent. Following the inhibition of angiogenesis, metastasis of the primary tumor is affected $(10,11)$. Therefore, it is necessary to investigate novel factors that may inhibit metastasis by affecting angiogenesis in human PTC.

Pigment epithelium-derived factor (PEDF) is a $50-\mathrm{kDa}$ secreted glycoprotein that was initially identified in cultured retinal pigment epithelial cells (12). PEDF has numerous biological functions, including differentiating activity, neurite outgrowth, survival activity, anti-apoptosis and anti-angiogenic activities and the induction of cell death (13-19). The anticancer role of PEDF has yet to be elucidated; however, previous studies have suggested various roles for PEDF in inhibiting cancer progression. For instance, PEDF may cause tumors to differentiate to a less malignant phenotype, PEDF may block angiogenesis-mediated activities and neovascularization, and PEDF may suppress tumor cell invasion and metastasis $(14,15)$. 
Table I. Information for the primers used in the quantitative polymerase chain reaction.

Gene

Sequence of primers (5'-3')

Length of target fragment (bp)

\begin{tabular}{ll}
\hline GAPDH & Forward: CCACATCGCTCAGACACCAT \\
& Reward: AGTTGAGGTCAATGAAGGGGT \\
PEDF & Forward: CTCGCCATGAGATCAGCATTC \\
& Reward: AGCCATAGCGTAAAACAGCCT \\
$V E G F$ & Forward: CTCGCCATGAGATCAGCATTC \\
& Reward: AGCCATAGCGTAAAACAGCCT \\
HIFla & Forward: TGTCGGAGTTTGGAAAACAA \\
& Reward: AAGTGGCAACTGATGAGCAA
\end{tabular}

$P E D F$, pigment epithelium-derived factor; $V E G F$, vascular endothelial growth factor; $H I F 1 \alpha$, hypoxia-inducible factor $1 \alpha$.

PEDF is one of the most promising anti-angiogenic factors; a number of studies have demonstrated its anti-angiogenic effects in various tumor models, including retinoblastoma, neuroblastoma, prostate cancer, melanoma, Wilms' tumor, pancreatic adenocarcinoma, hepatoblastoma, osteosarcoma, chondrosarcoma, human cervical carcinoma, gastric carcinoma, nasopharyngeal carcinoma, Lewis lung carcinoma, colorectal peritoneal carcinoma, glioma and breast cancer xenografts (20-24). The anti-angiogenic effect of PEDF is performed primarily through the disruption of microvascular network distribution (25-28). Vascular endothelial growth factor (VEGF) is an established pro-angiogenic factor and numerous studies have reported an inverse correlation between PEDF and VEGF expression levels in certain tumor models $(21,25-28)$.

The degree of oxygenation is crucial to neovascularization (29). The transcription factor hypoxia-inducible factor $1 \alpha$ (HIF1 $\alpha$ ) is a critical protein involved in the response to hypoxia, and is able to activate several downstream factors, including VEGF and glucose transporter 1; its expression is associated with tumor progression in various carcinomas, including pancreatic cancer, breast cancer, cervical carcinoma and thyroid cancer (29-31). Considering the associations between PEDF and tumor progression, PEDF expression levels in patients with PTC were evaluated in the current study. To the best of our knowledge, the present study is the first to investigate the association between PEDF expression levels and aggressive clinicopathological features in PTC, and to determine whether PEDF affects the lymph node metastasis (LNM) process in PTC by altering the HIF1 $\alpha$-VEGF signaling pathway.

\section{Materials and methods}

Patients. Patients with PTC who underwent thyroid excision surgery during the period 2011-2013 at The Second Affiliated Hospital of Harbin Medical University (Harbin, China) were recruited for the present study. The standard pathological diagnosis of PTC was based on the World Health Organization criteria (32) and two pathologists independently reviewed histological specimens in a blinded manner. A total of 271 patients with PTC ( 24 males and 247 females) were diagnosed and included in the current study. All patients met the inclusion criteria, which were: i) Underwent thyroid excision surgery; ii) confirmed as PTC by intraoperative rapid pathology and postoperative pathological detection; and iii) no history of thyroid disease and thyroid-related medication use. Informed consent was obtained from all participants and the study was performed according to the guidelines of the Ethics Committee of the Harbin Medical University.

Relevant patient data were collected, including demographic features (gender and age), clinical features (thyroid-stimulating hormone levels, tumor size and Hashimoto's disease) and pathological features (multifocality, $\mathrm{T}$ stage, extrathyroid invasion, intact capsule, prophylactic central compartment lymph-node (neck) dissection and node metastases). Tumor-node-metastasis (TNM) classification was performed according to the American Joint Committee on Cancer (33). T1 was defined as a tumor $\leq 2 \mathrm{~cm}$ in diameter and limited to the thyroid gland. T2 was defined as a tumor that was $>2$ and $\leq 4 \mathrm{~cm}$, and limited to the thyroid gland. T3 was defined as a tumor $>4 \mathrm{~cm}$ and limited to the thyroid gland, or any tumor with minimal glandular infiltration, including infiltration of the sternothyroid muscle or perithyroid soft tissues.

Immunohistochemical analysis. PTC tissue specimens were sectioned $(4-\mu \mathrm{m})$, deparaffinized in xylene and rehydrated in a graded series of ethanol. The slides were then incubated in $3 \%$ hydrogen peroxide in distilled water at room temperature for $10 \mathrm{~min}$ to inactivate endogenous peroxidase activity, and subsequently underwent antigen retrieval in a sodium citrate solution in a microwave oven. The tissue sections were blocked with 5\% bovine serum albumin (Boster Bio-Engineering, Wuhan, China) for $30 \mathrm{~min}$ at room temperature, and then incubated overnight at $4^{\circ} \mathrm{C}$ with a rabbit anti-human PEDF primary polyclonal antibody at 1:100 dilution (\#BA1348-1; Boster Bio-Engineering) in a humidified chamber. The tissue sections were then incubated with a ready-to-use biotinylated horseradish-peroxidase-conjugated secondary antibody (\#SA1022; Boster Bio-Engineering) and a streptavidin biotin complex (\#SA1022; Boster Bio-Engineering) at $37^{\circ} \mathrm{C}$ for $30 \mathrm{~min}$. The staining procedures were performed according to the manufacturer's instructions: Visualization with a 3,3'-diaminobenzidine solution and counterstaining with hematoxylin. The distribution and expression levels of PEDF were examined from the images obtained using the Olympus Imaging system (DP73: Olympus Corporation, Tokyo, Japan). 
Table II. Clinicopathological characteristics of patients with papillary thyroid carcinoma, recruited between 2011 and 2013.

\begin{tabular}{|c|c|}
\hline Characteristic & Value \\
\hline \multicolumn{2}{|l|}{ Gender } \\
\hline Male & $24(8.9)$ \\
\hline Female & $247(91.1)$ \\
\hline Age, years & $43.1 \pm 10.6(19-73)$ \\
\hline$<45$ & $154(56.8)$ \\
\hline$\geq 45$ & $117(43.2)$ \\
\hline Tumor size, mm & $10.9 \pm 7.8(2-50)$ \\
\hline \multicolumn{2}{|l|}{ Multifocality } \\
\hline Single & $259(95.6)$ \\
\hline Multiple ( $\geq 2)$ & $12(4.4)$ \\
\hline \multicolumn{2}{|l|}{ Extrathyroid invasion } \\
\hline Negative & $257(94.8)$ \\
\hline Positive & $14(5.2)$ \\
\hline \multicolumn{2}{|l|}{ Lymph node metastases } \\
\hline Negative & $185(68.3)$ \\
\hline Positive & $86(31.7)$ \\
\hline \multicolumn{2}{|l|}{ Hashimoto's disease } \\
\hline Negative & $226(83.4)$ \\
\hline Positive & $45(16.6)$ \\
\hline \multicolumn{2}{|l|}{ TNM stage } \\
\hline $\mathrm{T} 1$ & $245(90.4)$ \\
\hline $\mathrm{T} 3$ & $26(9.6)$ \\
\hline \multicolumn{2}{|l|}{$\mathrm{BRAF}^{\mathrm{V} 600 \mathrm{E}}$ mutation } \\
\hline Negative & $81(29.9)$ \\
\hline Positive & $190(70.1)$ \\
\hline \multicolumn{2}{|l|}{ PEDF } \\
\hline Negative & $69(25.5)$ \\
\hline Weakly positive & $131(48.3)$ \\
\hline Moderately positive & $71(26.2)$ \\
\hline
\end{tabular}

Data are presented as $\mathrm{n}(\%)$ or mean \pm standard deviation (range). TNM, tumor-node-metastasis; PEDF, pigment epithelium-derived factor; BRAF, B-rapidly accelerated fibrosarcoma.

PEDF expression levels were semi quantitatively categorized into three groups, as follows: Negative ( 0 points), $\leq 5 \%$ positive cells; weakly positive (1 point), 6-30\% positive cells; moderately positive ( 2 points), $31-60 \%$ positive cells.

Laser-capture microdissection. The frozen tissues specimens obtained from PTC patients with LNM $(n=15)$ and without LNM ( $n=10)$ were used for laser capture microdissection (ArcturusXT ${ }^{\mathrm{TM}}$; Thermo Fisher Scientific, Inc., Waltham, MA, USA) to obtain target thyroid epithelial cells, as described previously (34). The age and gender of the participants were matched for each group.

Reverse transcription-quantitative polymerase chain reaction $(R T-q P C R)$. Total RNA was extracted from the microdissected cells using RNAiso Plus (9108; Takara Biotechnology Co., Ltd.,

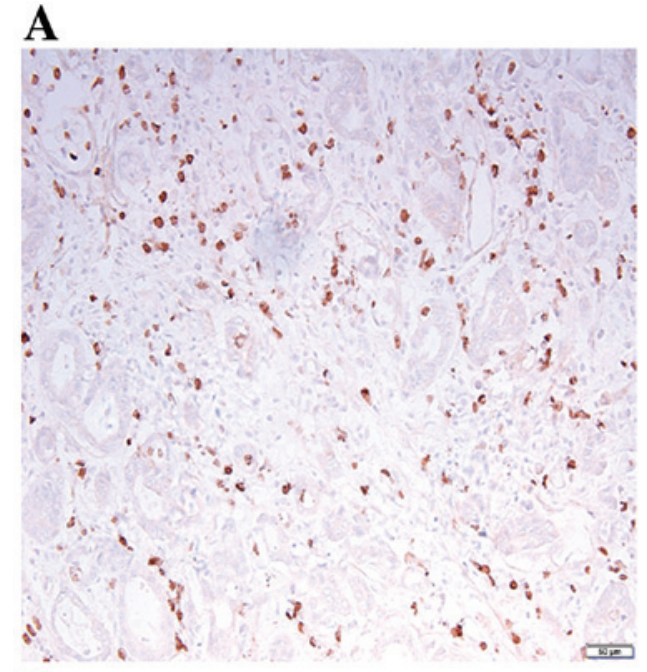

B

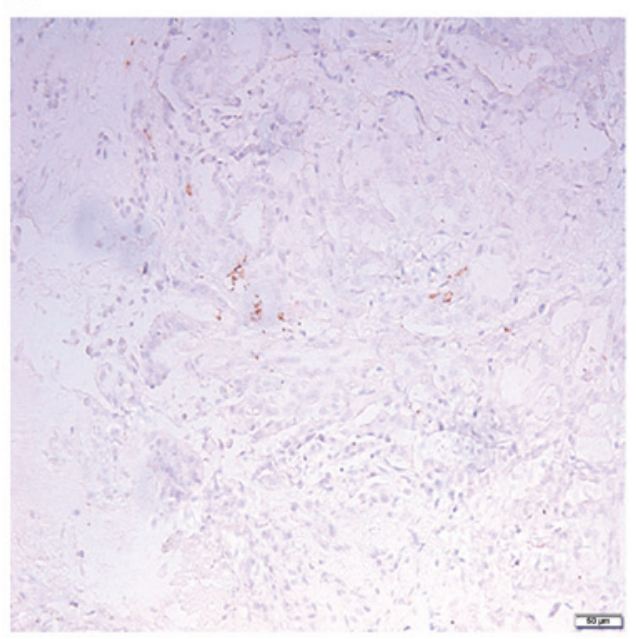

Figure 1. Typical immunohistochemical staining for PEDF (magnification, x200). (A) The LNM positive group. (B) The LNM negative group. PEDF, pigment epithelium-derived factor; LNM, lymph node metastasis.

Dalian, China). The extracted RNA was subsequently incubated with Recombinant DNase I (D2270; Takara Biotechnology Co., Ltd.) to erase the genomic DNA. cDNA was then obtained from mRNA using the PrimeScript ${ }^{\mathrm{TM}}$ RT reagent kit (DRR025A; Takara Biotechnology Co., Ltd.), which was then amplified using PCR on the ABI 7500 Real-Time PCR system (Thermo Fisher Scientific, Inc.) with SYBR Green I dye (DRR041S; Takara Biotechnology Co., Ltd.), in accordance with the manufacturer's protocol. The cycling conditions were as follows: 1 cycle as an initial denaturation at $95^{\circ} \mathrm{C}$ for $10 \mathrm{~min} ; 40$ cycles of $95^{\circ} \mathrm{C}$ for $5 \mathrm{sec}, 60^{\circ} \mathrm{C}$ for $30 \mathrm{sec}$ and $72^{\circ} \mathrm{C}$ for $15 \mathrm{sec}$; and a final extension step at $72^{\circ} \mathrm{C}$ for $5 \mathrm{~min}$. The relative expression levels of PEDF, VEGF and HIFl $\alpha$ were determined using the comparative $\mathrm{Cq}$ method (35), following normalization to the endogenous GAPDH control gene. The primer sequences used in the RT-qPCR are presented in Table I.

Statistical analysis. All statistical analyses in the current study were performed with SPSS software, version 13.01S (SPSS, Inc., Chicago, IL, USA). An independent-sample $t$-test was used to compare the means between two groups, and a $\chi^{2}$ or Fisher's exact test was used to compare frequencies between 
Table III. Correlation between patient clinicopathological features and PEDF expression levels.

\begin{tabular}{|c|c|c|c|c|}
\hline Characteristic & Negative & Weakly positive & Moderately positive & P-value \\
\hline Gender & & & & 0.939 \\
\hline Male & $63(91.3)$ & $120(91.6)$ & $64(90.1)$ & \\
\hline Female & $6(8.7)$ & $11(8.4)$ & $7(9.9)$ & \\
\hline Age, years & & & & 0.878 \\
\hline$<45$ & $41(59.4)$ & $73(55.7)$ & $40(56.3)$ & \\
\hline$\geq 45$ & $28(40.6)$ & $58(44.3)$ & $31(43.7)$ & \\
\hline Tumor size, mm & & & & 0.018 \\
\hline$\leq 10$ & $33(47.8)$ & $75(57.3)$ & $48(67.6)$ & \\
\hline$>10$ & $36(52.2)$ & $56(42.7)$ & $23(32.4)$ & \\
\hline Multifocality & & & & 0.152 \\
\hline Single & $62(91.2)$ & $127(96.9)$ & $70(97.2)$ & \\
\hline Multiple & $6(8.8)$ & $4(3.1)$ & $2(2.8)$ & \\
\hline Extrathyroid invasion & & & & 0.035 \\
\hline Negative & $61(88.4)$ & $127(96.9)$ & $69(97.2)$ & \\
\hline Positive & $8(11.6)$ & $4(3.1)$ & $2(2.8)$ & \\
\hline Lymph node metastasis & & & & 0.006 \\
\hline Negative & $41(59.4)$ & 85 (64.9) & $59(83.1)$ & \\
\hline Positive & $28(40.6)$ & $46(35.1)$ & $12(16.9)$ & \\
\hline TNM stage & & & & 0.013 \\
\hline $\mathrm{T} 1$ & $57(82.6)$ & $119(90.8)$ & $69(97.2)$ & \\
\hline T3 & $12(17.4)$ & $12(9.2)$ & $2(2.8)$ & \\
\hline $\mathrm{BRAF}^{\mathrm{V} 600 \mathrm{E}}$ mutation & & & & $<0.001$ \\
\hline Negative & $8(11.6)$ & $33(25.2)$ & $40(56.3)$ & \\
\hline Positive & $61(88.4)$ & $98(74.8)$ & $31(43.7)$ & \\
\hline
\end{tabular}

Data are presented as n (\%). TNM, tumor-node-metastasis; PEDF, pigment epithelium-derived factor; BRAF, B-rapidly accelerated fibrosarcoma

Table IV. Correlation analysis among the mRNA expression levels of PEDF, VEGF and HIF $1 \alpha$.

\begin{tabular}{|c|c|c|}
\hline Analysis & Correlation coefficient (r) & P-value \\
\hline$P E D F$ vs. VEGF & -0.514 & 0.009 \\
\hline$P E D F$ vs. $H I F l \alpha$ & -0.287 & 0.164 \\
\hline$V E G F$ vs. $H I F l \alpha$ & 0.489 & 0.013 \\
\hline
\end{tabular}

$P E D F$, pigment epithelium-derived factor; $V E G F$, vascular endothelial growth factor; HIFl $\alpha$, hypoxia-inducible factor $1 \alpha$.

the groups. The data are presented as the mean \pm standard deviation, or as percentages where appropriate. A Pearson correlation analysis was used to analyze the associations among the expression levels of PEDF, VEGF and HIF $1 \alpha$ in PTC cells. All $t$-tests were two-tailed; $\mathrm{P}<0.05$ was considered to indicate a statistically significant difference.

\section{Results}

Clinicopathological characteristics of patients with PTC. The clinicopathological characteristics of the patients are shown

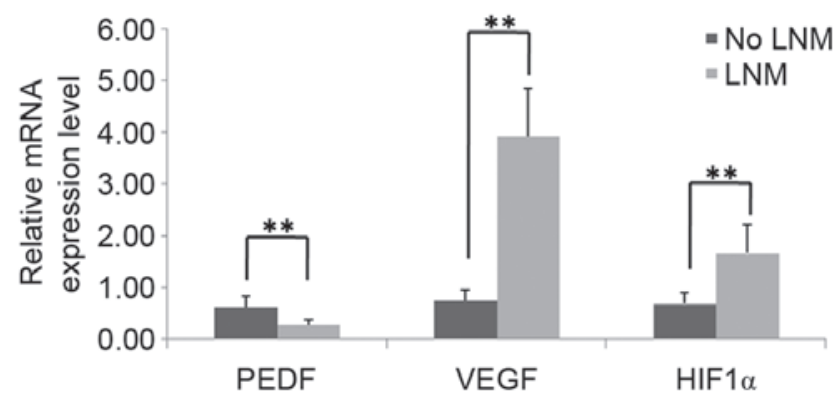

Figure 2. Reverse transcription-quantitative polymerase chain reaction analysis of PEDF, VEGF and HIF $1 \alpha$ mRNA expression levels. ${ }^{* *} \mathrm{P}<0.01$. PEDF, pigment epithelium-derived factor; $V E G F$, vascular endothelial growth factor; HIFl $\alpha$, hypoxia-inducible factor $1 \alpha$; LNM, lymph node metastasis.

in Table II. Between 2011 and 2013, 271 patients with PTC, including 24 male and 247 female patients, were enrolled in the present study. The mean age of the patients was $43.1 \pm 10.6$ years (age range, 19-73 years) and the mean size of the tumor was $10.9 \pm 7.8 \mathrm{~mm}$ (range, 2-50 mm). Multifocal tumors were identified in 12 patients; 14 patients $(5.2 \%)$ had extrathyroid invasion and 86 patients $(31.7 \%)$ had LNM. Hashimoto's disease was observed in 45 patients. A total of 245 patients had T1 tumors, 26 had T3 tumors and none had tumors in another stage. The 
$\mathrm{BRAF}^{\mathrm{V} 600 \mathrm{E}}$ mutation was present in $70.1 \%$ of patients. Immunohistochemistry with an anti-PEDF antibody detected PEDF expression in $74.5 \%$ of the PTC tissues. PEDF expression was determined to be negative in 69 patients, weakly positive in 131 patients and moderately positive in 71 patients.

Decreased PEDF levels correlate with the progression of human PTC. In order to determine whether PEDF contributes to the progression of PTC, the associations between PEDF and aggressive clinicopathological features were investigated. As a result of the importance of metastasis to the progression of PTC, the association between PEDF expression levels and LNM was initially evaluated. As presented in Fig. 1 and Table III, PEDF expression levels were significantly decreased in PTC tissues with LNM, as compared with PTC tissues without LNM $(\mathrm{P}=0.006)$. Similarly, PEDF expression levels were significantly decreased in PTC tissues with extrathyroid invasion $(\mathrm{P}=0.035)$, a high TNM stage $(\mathrm{P}=0.013), \mathrm{BRAF}^{\mathrm{V} 600 \mathrm{E}}$ mutation positivity $(\mathrm{P}<0.001)$ and a tumor size of $>10 \mathrm{~mm}$ $(\mathrm{P}=0.018)$. However, PEDF expression levels were not significantly associated with multifocality ( $\mathrm{P}=0.152$; Table III).

Decreased expression levels of PEDF are accompanied by an upregulation of the HIF $1 \alpha-V E G F$ signaling pathway in patients with PTC and LNM. To elucidate the potential mechanisms underlying the role of PEDF in metastasis by affecting angiogenesis, the mRNA expression profiles of PEDF, VEGF and $H I F l \alpha$ in stored patient tissue samples were determined using RT-qPCR. As indicated in Fig. 2, PEDF mRNA expression levels were significantly decreased in PTC tissues with LNM (0.2766 \pm 0.0910$)$, as compared with PTC tissues without LNM $(0.6251 \pm 0.2034 ; \mathrm{P}<0.01)$. By contrast, the mRNA expression levels of $H I F l \alpha$ and $V E G F$ were significantly increased in PTC tissues with LNM (1.6646 \pm 0.5533 and 3.9321 \pm 0.9235 , respectively), as compared with PTC tissues without LNM (0.6847 \pm 0.2240 and $0.7537 \pm 0.1988$, respectively; $\mathrm{P}<0.01)$. In addition, as shown in Table IV, through the analysis of the mRNA expression levels of PEDF, VEGF and HIFl $\alpha$ in the thyroid tissues specimens obtained from PTC patients with LNM $(n=15)$ and without LNM $(n=10)$, the present study identified a significant inverse correlation between $P E D F$ and $V E G F$ expression levels ( $\mathrm{r}=-0.514 ; \mathrm{P}=0.009)$, an inverse association between PEDF and HIFl $\alpha$ expression levels ( $\mathrm{r}=-0.287$; $\mathrm{P}=0.164)$ and a significant positive correlation between $V E G F$ and HIFl $\alpha$ expression levels $(\mathrm{r}=0.489 ; \mathrm{P}=0.013)$.

\section{Discussion}

To the best of our knowledge, the present study is the first to demonstrate that PEDF expression levels are correlated with specific patient clinicopathological features, including LNM, extrathyroid invasion, $\mathrm{BRAF}^{\mathrm{V} 600 \mathrm{E}}$ mutation positivity, tumor size and a high TNM stage in PTC tissues. The results also indicated that PEDF may have an important role in metastasis by affecting angiogenesis induced by HIF1 $\alpha$.

The actions of PEDF have been evaluated in various types of tumor cells. The results of a number of previous studies support the hypothesis that PEDF expression may promote tumor growth, invasion and metastasis $(19,21,22,25,26)$. Reduced PEDF expression is a potent promoter of tumor growth and angiogenesis in breast cancer (36), and the loss of PEDF enables melanoma cells to acquire an invasive phenotype (37). The results of the present study were concordant with previous findings in the majority of solid tumors $(22,26,36,37)$, which suggested that PEDF may have an important role in the progression of PTC. However, certain observations of the current study, including the association of PEDF expression levels with tumor stage and its lack of association with multifocality, must be investigated in further studies with a larger cohort, as only 26 cases of a T3 tumor and 12 cases of multifocality were recruited for the present study.

Considering its potent function in angiogenesis, it was hypothesized that PEDF exerts its role in metastasis mainly via affecting angiogenesis in PTC tissues. Previous studies have demonstrated that the HIF1 $\alpha$-VEGF signaling pathway is activated during the progression of thyroid cancer $(38,39)$. To the best of our knowledge, the present study is the first to identify an inverse association between PEDF expression levels and the HIF1 $\alpha$-VEGF signaling pathway in human PTC tissues. Recent studies supported these findings. For instance, PEDF was able to suppress angiogenesis in a gastric carcinoma xenograft model by down regulating HIF1 $\alpha$ and VEGF expression (27). Furthermore, PEDF suppressed tumor growth in a cervical cancer cell line by down regulating the expression of VEGF and HIF1 $\alpha$, which demonstrated the anti-angiogenic activity of PEDF (26). In addition, the HIF1 $\alpha$ and VEGF/PEDF signaling pathway is targeted in impacting nasopharyngeal carcinoma cell proliferation and angiogenesis (28). PEDF may not be directly regulated by HIF1 $\alpha$, as indicated by the inverse association between PEDF and HIF1 $\alpha$ identified in the current study. Therefore, HIF1 $\alpha$ may also regulate the expression of PEDF via other factors in human PTC, which requires further analysis.

In conclusion, the results of the current study suggested that PEDF expression levels were significantly associated with certain aggressive clinicopathological features in human PTC, and indicated that PEDF may exert an anti-angiogenesis role by modulating the HIF1 $\alpha$-VEGF signaling pathway, which has an important role in the metastasis of PTC. Additional studies should further investigate these results using in vitro and in vivo models, and elucidate the mechanism underlying the function of PEDF in anti-angiogenesis, through the knock down of the PEDF gene.

\section{Acknowledgements}

This study was supported by the Natural Science Foundation of Heilongjiang Province of China (grant no. D201228).

\section{References}

1. Udelsman $\mathrm{R}$ and Chen $\mathrm{H}$ : The current management of thyroid cancer. Adv Surg 33: 1-27, 1999.

2. Paterson IC, Greenlee R and Adams Jones D: Thyroid cancer in Wales, 1985-1996: A cancer registry-based study. Clin Oncol (R Coll Radiol) 44: 245-251, 1999.

3. Davies L and Welch HG: Increasing incidence of thyroid cancer in the United States, 1973-2002. JAMA 295: 2164-2167, 2006.

4. De Lellis R, Lloyd R, Heitz PU and Eng C: Pathology and genetics of tumors of the endocrine organs. IARC, Lyon, 2004.

5. Mazzaferri EL and Jhiang SM: Long-term impact of initial surgical and medical therapy on papillary and follicular thyroid cancer. Am J Med 97: 418-428, 1994.

6. Schlumberger MJ: Papillary and follicular thyroid carcinoma. N Engl J Med 338: 297-306, 1998. 
7. Desgrosellier JS and Cheresh DA: Integrins in cancer: Biological implications and therapeutic opportunities. Nat Rev Cancer 10: 9-22, 2010.

8. Grivennikov SI, Greten FR and Karin M: Immunity, inflammation, and cancer. Cell 140: 883-899, 2010.

9. Kohn EC and Liotta LA: Molecular insights into cancer invasion: Strategies for prevention and intervention. Cancer Res 55: 1856-1862, 1995.

10. Nucera C, Lawler J and Parangi S: BRAF(V600E) and microenvironment in thyroid cancer: A functional link to drive cancer progression. Cancer Res 71: 2417-2422, 2011.

11. Li Y, Zhai Z, Liu D, Zhong X, Meng X, Yang Q, Liu J and Li H: CD105 promotes hepatocarcinoma cell invasion and metastasis through VEGF. Tumour Biol 36: 737-745, 2015.

12. Tombran-Tink J, Chader GG and Johnson LV: PEDF: A pigment epithelium-derived factor with potent neuronal differentiative activity. Exp Eye Res 53: 411-414, 1991.

13. Becerra SP and Notario V: The effects of PEDF on cancer biology: Mechanisms of action and therapeutic potential. Nat Rev Cancer 13: 258-271, 2013.

14. Filleur S, Volz K, Nelius T, Mirochnik Y, Huang H, Zaichuk TA Aymerich MS, Becerra SP, Yap R, Veliceasa D, et al: Two functional epitopes of pigment epithelial-derived factor block angiogenesis and induce differentiation in prostate cancer. Cancer Res 65: 5144-5152, 2005.

15. Crawford SE, Stellmach V, Ranalli M, Huang X, Huang L, Volpert O, De Vries GH, Abramson LP and Bouck N: Pigment epithelium-derived factor (PEDF) in neuroblastoma: A multifunctional mediator of Schwann cell antitumor activity. J Cell Sci 114 4421-4428, 2001.

16. Tanimoto S, Kanamoto T, Mizukami M, Aoyama H and Kiuchi Y: Pigment epithelium-derived factor promotes neurite outgrowth of retinal cells. Hiroshima J Med Sci 55: 109-116, 2006.

17. Unterlauft JD,Eichler W,KuhneK, Yang XM, Yafai Y,WiedemannP, Reichenbach A and Claudepierre T: Pigment epithelium-derived factor released by Muller glial cells exerts neuroprotective effects on retinal ganglion cells. Neurochem Res 37: 1524-1533, 2012

18. Cao W, Tombran-Tink J, Chen W, Mrazek D, Elias R and McGinnis JF: Pigment epithelium-derived factor protects cultured retinal neurons against hydrogen peroxide -induced cell death. J Neurosci Res 57: 789-800, 1999.

19. Dawson DW, Volpert OV, Gillis P, Crawford SE, Xu H, Benedict W and Bouck NP: Pigment epithelium-derived factor: A potent inhibitor of angiogenesis. Science 285: 245-248, 1999.

20. Stellmach V, Crawford SE, Zhou W and Bouck N: Prevention of ischemia-induced retinopathy by the natural ocular antiangiogenic agent pigment epithelium-derived factor. Proc Natl Acad Sci USA 98: 2593-2597, 2001.

21. Browne M, Stellmach V, Cornwell M, Chung C, Doll JA, Lee EJ, Jameson JL, Reynolds M, Superina RA, Abramson LP and Crawford SE: Gene transfer of pigment epithelium-derived factor suppresses tumor growth and angiogenesis in a hepatoblastoma xenograft model. Pediatr Res 60: 282-287, 2006.

22. Garcia M, Fernandez-Garcia NI, Rivas V, Carretero M, Escamez MJ, Gonzalez-Martin A, Medrano EE, Volpert O, Jorcano JL, Jimenez B, et al: Inhibition of xenografted human melanoma growth and prevention of metastasis development by dual antiangiogenic/antitumor activities of pigment epithelium-derived factor. Cancer Res 64: 5632-5642, 2004

23. Wu QJ, Gong CY, Luo ST, Zhang DM, Zhang S, Shi HS, Lu L, Yan HX, He SS, Li DD, et al: AAV-mediated human PEDF inhibits tumor growth and metastasis in murine colorectal peritoneal carcinomatosis model. BMC Cancer 12: 129, 2012.

24. Maik-Rachline G, Shaltiel S and Seger R: Extracellular phosphorylation converts pigment epithelium-derived factor from a neurotrophic to an antiangiogenic factor. Blood 105: 670-678, 2005
25. Yang H, Cheng R, Liu G, Zhong Q, Li C, Cai W, Yang Z, Ma J, Yang $\mathrm{X}$ and Gao G: PEDF inhibits growth of retinoblastoma by anti-angiogenic activity. Cancer Sci 2100: 2419-2425, 2009.

26. Yang J, Chen S, Huang X, Han J, Wang Q, Shi D, Cheng R, Gao $\mathrm{G}$ and Yang X: Growth suppression of cervical carcinoma by pigment epithelium-derived factor via anti-angiogenesis. Cancer Biol Ther 9: 967-974, 2010.

27. Zhang Y, Han J, Yang X, Shao C, Xu Z, Cheng R, Cai W, Ma J, Yang $\mathrm{Z}$ and Gao G: Pigment epithelium-derived factor inhibits angiogenesis and growth of gastric carcinoma by down-regulation of VEGF. Oncol Rep 26: 681-686, 2011.

28. Xu Z, Fang S, Zuo Y, Zhang Y, Cheng R, Wang Q, Yang Z, Cai W, Ma J, Yang X and Gao G: Combination of pigment epithelium-derived factor with radiotherapy enhances the Anti-tumor effects on nasopharyngeal carcinoma by down-regulating vascular endothelial growth factor expression and angiogenesis. Cancer Sci 102: 1789-1798, 2011.

29. Birner P, Schindl M, Obermair A, Plank C, Breitenecker G and Oberhuber G: Overexpression of hypoxia-inducible factor 1alpha is a marker for an unfavorable prognosis in early-stage invasive cervical cancer. Cancer Res 60: 4693-4696, 2000.

30. Schindl M, Schoppmann SF, Samonigg H, Hausmaninger H, Kwasny W, Gnant M, Jakesz R, Kubista E, Birner P and Oberhuber G; Austrian Breast and Colorectal Cancer Study Group: Overexpression of hypoxia-inducible factor 1alpha is associated with an unfavorable prognosis in lymph node-positive breast cancer. Clin Cancer Res 8: 1831-1837, 2002.

31. Koperek O, Akin E, Asari R, Niederle B and Neuhold N: Expression of hypoxia-inducible factor 1 alpha in papillary thyroid carcinoma is associated with desmoplastic stromal reaction and lymph node metastasis. Virchows Arch 463: 795-802, 2013

32. Hedinger C, Williams ED and Sobin LH: The WHO histological classification of thyroid tumors: A commentary on the second edition. Cancer 63: 908-911, 1989.

33. Stratmann M, Sekulla C, Dralle H and Brauckhoff M: Current TNM system of the UICC/AJCC: The prognostic significance for differentiated thyroid carcinoma. Chirurg 83: 646-651, 2012 (In German).

34. Wang L, Liu R, Li W, Chen C, Katoh H, Chen GY, McNally B, Lin L, Zhou P, Zuo T, et al: Somatic single hits inactivate the $\mathrm{X}$-linked tumor suppressor FOXP3 in the prostate. Cancer Cell 16: 336-346, 2009

35. Livak KJ and Schmittgen TD: Analysis of relative gene expression data using real-time quantitative PCR and the 2(-Delta Delta C(T)) Method. Methods 25: 402-408, 2001.

36. Cai J, Parr C, Watkins G, Jiang WG and Boulton M: Decreased pigment epithelium-derived factor expression in human breast cancer progression. Clinical Cancer Res 12: 3510-3517, 2006.

37. Orgaz JL, Ladhani O, Hoek KS, Fernández-Barral A, Mihic D, Aguilera O, Seftor EA, Bernad A, Rodríguez-Peralto JL, Hendrix MJ, et al: Loss of pigment epithelium-derived factor enables migration, invasion and metastatic spread of human melanoma. Oncogene 28: 4147-4161, 2009.

38. Lan L, Luo Y, Cui D, Shi BY, Deng W, Huo LL, Chen HL, Zhang GY and Deng LL: Epithelial-mesenchymal transition triggers cancer stem cell generation in human thyroid cancer cells. Int J Oncol 43: 113-120, 2013.

39. Koperek O, Bergner O, Pichlhöfer B, Oberndorfer F, Hainfellner JA, Kaserer K, Horvat R, Harris AL, Niederle B and Birner P: Expression of hypoxia-associated proteins in sporadic medullary thyroid cancer is associated with desmoplastic stroma reaction and lymph node metastasis and may indicate somatic mutations in the VHL gene. J Pathol 225: 63-72, 2011. 